DCPT-12/05

\title{
Derivation of the blackfold effective theory
}

\author{
Joan $\operatorname{Camps}^{a}$, Roberto Emparan ${ }^{b, c}$ \\ a Centre for Particle Theory $\&$ Department of Mathematical Sciences \\ Science Laboratories, South Road, Durham DH1 3LE, United Kingdom \\ ${ }^{b}$ Institució Catalana de Recerca i Estudis Avançats (ICREA) \\ Passeig Lluís Companys 23, E-08010 Barcelona, Spain \\ ${ }^{c}$ Departament de Física Fonamental and \\ Institut de Ciències del Cosmos, Universitat de Barcelona, \\ Martí i Franquès 1, E-08028 Barcelona, Spain \\ joan.camps@durham.ac.uk, emparan@ub.edu
}

\begin{abstract}
We study fluctuations and deformations of black branes over length scales larger than the horizon radius. We prove that the Einstein equations for the perturbed $p$ brane yield, as constraints, the equations of the effective blackfold theory. We solve the Einstein equations for the perturbed geometry and show that it remains regular on and outside the black brane horizon. This study provides an ab initio derivation of the blackfold effective theory and gives explicit expressions for the metrics near the new black holes and black branes that result from it, to leading order in a derivative expansion.
\end{abstract}




\section{Introduction}

The blackfold approach is a worldvolume effective theory for the dynamics of black branes [1, 2]. When the worldvolume of the brane is spatially compact, it provides an efficient tool to study new higher-dimensional black holes in regimes in which their horizons possess two widely separated length scales [3]. It has also proved useful for understanding the stability of black holes and black branes under long-wavelength perturbations [2, 4].

This effective theory captures the dynamics of a black brane when it deviates away from the flat uniform state over a scale $R$ much longer than the brane thickness $r_{0}$. This can happen either because the brane becomes inhomogenous, with thickness varying along the worldvolume (intrinsic fluctuations) or because the worldvolume bends in the background spacetime (extrinsic fluctuations). The dynamics of the brane is conveniently expressed in terms of its effective stress-energy tensor $T_{a b}$, which is computed in the region $r_{0} \ll r \ll R$ where the gravitational field is weak. In [2], using an argument based on general covariance, the effective theory equations were determined to be

$$
D_{a} T^{a b}=0
$$

for intrinsic fluctuations, where $D_{a}$ is the covariant derivative for the worldvolume metric, and

$$
K_{a b}^{\rho} T^{a b}=0,
$$

for extrinsic fluctuations, where $K_{a b}{ }^{\rho}$ is the extrinsic curvature tensor for the embedding of the brane worldvolume in the background spacetime. The equations (1.1) are of hydrodynamic type, while (1.2) are elasticity equations for a brane: black branes behave as liquids under strains parallel to their worldvolume, and like elastic solids under strains orthogonal to them.

These equations have been shown to describe correctly several properties of black holes and black branes that were previously known, and have also yielded new solutions and new insights. However, in order to more solidly ground the theory it is necessary to

1. Derive the blackfold equations (1.1) and (1.2) directly from the Einstein equations that describe a black $p$-brane with generic perturbations of length scale $R \gg r_{0}$.

2. Prove that the horizon of the black $p$-brane remains regular under these perturbations.

The purpose of this paper is to establish these two points. 
The problem is a more familiar one in the case of $p=0$. It is well known that, to the extent that a black hole can be seen as a point particle, it must follow a geodesic worldline [5]. There are two sorts of arguments for this: (i) general covariance and minimal coupling (i.e., lowest derivative order) imply that the equations of motion of the particle are uniquely fixed to be those of timelike geodesics; (ii) the Einstein equations for a trajectory that is a small perturbation of the static black hole solution yield the geodesic equation as a constraint, and then the remaining Einstein equations admit a unique solution that is regular on and outside the perturbed horizon. We may regard (i) as abstract generic derivations, and (ii) as 'microscopic' or ab initio derivations. The path to the blackfold equations in ref. [2] followed the abstract generic approach of (i). Our purpose here is to derive these equations in the more detailed manner of (ii). Moreover, just like geodesic motion describes the dynamics of any small particle, not necessarily a black hole, similarly the extrinsic equations (1.2) are also applicable for all thin $p$-branes, not only black ones 1 . Our proof of these equations will indeed have this generality. The study of regularity of the perturbed solution is instead theory-specific, in the present case to neutral black $p$-branes.

In the next section we set up our study of generic long wavelength perturbations, and argue that intrinsic and extrinsic perturbations decouple at the leading order in a derivative expansion. Intrinsic perturbations have been solved already in [4]. Then we proceed to set up the problem for extrinsic perturbations by constructing Fermi normal coordinates adapted to $p$-branes. In section 3 we derive the extrinsic equations (1.2) from the gravitational dynamics of a generic $p$-brane. In section 4 we solve the Einstein equations for a black $p$-brane under extrinsic perturbations. In section 5 we show that the horizon remains regular. Section 6 puts together in a covariant manner the results for the metric of a black brane under a generic long-wavelength perturbation. Section 7 contains a final discussion of our results. As in previous works, for a $p$-brane in $D$-dimensional spacetime we find convenient to introduce

$$
n=D-p-3 \text {. }
$$

\section{Set up}

\subsection{Long-wavelength perturbations of black branes}

We write the metric for a black $p$-brane in the form

$$
d s^{2}=\left(\eta_{a b}+\frac{r_{0}^{n}}{r^{n}} u_{a} u_{b}\right) d \sigma^{a} d \sigma^{b}+\frac{d r^{2}}{1-\frac{r_{0}^{n}}{r^{n}}}+r^{2} d \Omega_{(n+1)}^{2},
$$

\footnotetext{
${ }^{1}$ Eqs. (1.2) were first obtained from covariance arguments of type (i) in [6].
} 
where the coordinates $\sigma^{a}$ span the worldvolume of the $p$-brane with the Minkowski metric $\eta_{a b}, a, b=0, \ldots p$. We have introduced a constant worldvolume vector $u^{a}$ that characterizes the local worldvolume velocity and satisfies $\eta_{a b} u^{a} u^{b}=-1$. In this form, the parameters of the solution are the horizon radius $r_{0}$ and the components of the velocity $u^{a}$. In addition to these, there are $D-p-1$ coordinates for the position of the black brane in the transverse space, $X^{\perp}$, which in (2.1) are fixed to the origin. They can be made explicit by a shift of coordinates in the transverse directions, but this results in cumbersome expressions that we will not need.

Constant shifts of these parameters still give solutions to the Einstein equations. Our purpose is to describe solutions in which the parameters vary slowly along the worldvolume. In a manner patterned after [7], we write the modified geometry as

$$
\begin{aligned}
d s^{2}= & \left(\gamma_{a b}\left(X^{\mu}(\sigma)\right)+\frac{r_{0}^{n}(\sigma)}{r^{n}} u_{a}(\sigma) u_{b}(\sigma)\right) d \sigma^{a} d \sigma^{b}+\frac{d r^{2}}{1-\frac{r_{0}^{n}(\sigma)}{r^{n}}}+r^{2} d \Omega_{(n+1)}^{2} \\
& +h_{\mu \nu}(x) d x^{\mu} d x^{\nu}
\end{aligned}
$$

where $\gamma_{a b}\left(X^{\mu}(\sigma)\right)=g_{\mu \nu} \partial_{a} X^{\mu} \partial_{b} X^{\nu}$ is the metric induced on the worldvolume by the embedding $X^{\mu}(\sigma)$ in the background metric $g_{\mu \nu} 2$ The additional terms $h_{\mu \nu}$ are of the same order as the derivatives of $\ln r_{0}, u^{a}$ and $X^{\perp}$, and their presence is required in order to have a solution of Einstein's equations. One of our aims is to compute these corrections and prove that the horizon remains regular in the corrected geometry.

We shall work to first order in the derivative expansion. This means that, locally, at any given point in the worldvolume the fluctuations are small and we only keep terms linear in the perturbations. This local linearization of the perturbations is essential for our analysis, as it allows us to decouple the intrinsic fluctuations (i.e., of $r_{0}$ and $u^{a}$, and the intrinsic curvature of the metric) from the extrinsic fluctuations (of $X^{\perp}$ ), and therefore we can discuss them separately.

\section{$2.2 \quad$ Intrinsic perturbations}

The study of long-wavelength fluctuations of $r_{0}$ and $u^{a}$, keeping the worldvolume metric flat, has been done in [4]. It has been shown that, to first order in the derivative expansion, the Einstein constraint equations $R_{b}^{r}=0$ require that eqs. (1.1) be satisfied, where

$$
T^{a b}=\frac{\Omega_{(n+1)}}{16 \pi G} r_{0}^{n}\left(n u^{a} u^{b}-\eta^{a b}\right)
$$

is the stress-energy tensor of the black brane measured in the asymptotically flat region $r \rightarrow \infty$. This stress-energy tensor can be obtained from an ADM-type prescription [8], or equivalently, from the Brown-York quasilocal stress-energy tensor [9].

\footnotetext{
${ }^{2}$ The transverse coordinate dependence is made more explicit in section 2.3 .
} 
Since (2.3) can be regarded as the stress-energy tensor of a perfect fluid, eqs. (1.1) are hydrodynamic Euler equations. Ref. 4] then showed that, for any solution of these $(p+1)$-dimensional equations, a solution of the $D$-dimensional Einstein equations for $h_{\mu \nu}$ can be constructed to leading order in derivatives, such that the horizon of the perturbed black brane remains regular. Thus, the analysis in [4] provides proof of the intrinsic blackfold equations that describe fluctuations of $r_{0}$ and $u^{a}$, and of the regularity of the black brane solutions that result.

It remains now in (2.2) to analyze the deviations of the worldvolume metric $\gamma_{a b}$ from the flat metric $\eta_{a b}$. These deformations can be of two kinds: intrinsic or extrinsic. Intrinsic variations of the metric, which only involve quantities constructed out of the worldvolume metric $\gamma_{a b}$ and its intrinsic curvature, can appear due to background curvature or be induced by the worldvolume embedding. However, around any given point on the worldvolume we can choose Riemann normal coordinates so that the intrinsic curvature shows up only at second order in derivatives. Intrinsic metric fluctuations are therefore negligible to the order that we work in this paper.

The extrinsic curvature of the worldvolume is of first order in derivatives, and will be the main focus of this paper.

\subsection{Adapted coordinates for curved branes}

The bending of the brane in directions orthogonal to its worldvolume is characterized by its extrinsic curvature tensor. We study the case in which the typical extrinsic curvature radius $R$ is much larger than the brane thickness $r_{0}$. At any given point on the worldvolume, the black brane is slightly perturbed away from flatness by terms that are linear in the fluctuations of the transverse coordinates. The effect can be appropriately captured by employing a set of coordinates, analogous to the Fermi normal coordinates around the trajectory of a particle, that describe the neighbourhood of a $(p+1)$-dimensional submanifold $\mathcal{W}_{p+1}$ of arbitrary codimension.

Fermi normal coordinates employ the idea, common to all normal coordinates, of eliminating first derivatives of the metric around a given point, but they are constrained to being adapted to a worldvolume that is not geodesically embedded in the background spacetime. Thus, these coordinates employ geodesic grids only in directions normal to $\mathcal{W}_{p+1}$. Some of the first derivatives of the metric are not eliminated, as they correspond to tensors that characterize the shape of the embedding of the worldvolume.

As explained above, to first order in derivatives one can choose normal coordinates $\sigma^{a}$ on $\mathcal{W}_{p+1}$ such that the connection on the worldvolume is manifestly flat, $\Gamma_{a b}^{c}=0$. We denote by $y^{i}$ the coordinates in the orthogonal directions, in such a way that $\mathcal{W}_{p+1}$ 
sits at $y^{i}=0$. Then

$$
d s^{2}=\eta_{a b} d \sigma^{a} d \sigma^{b}+d y_{i} d y^{i}+O(y / R)+O\left(\sigma^{2} / R_{i n t}^{2}\right)
$$

Transverse indices $i, j=p+1, \ldots, D$ are raised, lowered and contracted using the flat metric $\delta_{i j}$. We have introduced two characteristic lengths: $R$ for the extrinsic curvature radius, and $R_{\text {int }}$ for the intrinsic curvature radius of the worldvolume. Gauss-Codaccitype equations [10] imply that typically $R_{\text {int }} \sim R$. In the following we will omit to specify that the expansion is valid up to terms $O\left(\sigma^{2} / R_{\text {int }}^{2}\right)$.

Now we extend these coordinates away from the submanifold to include terms of first order in $y / R$. The Fermi construction adapted to the worldvolume of a $p$-brane assigns the coordinates $\left(\sigma^{a}, y^{i}\right)$ to the point that is reached by moving a unit affine parameter along the geodesic with tangent $\partial / \partial y^{i}$ that intersects $\mathcal{W}_{p+1}$ orthogonally at $\sigma^{a}$. Since $y^{i}$ now parametrize geodesics, we have

$$
\Gamma_{i j}^{\mu}=0, \quad \mu=a, i
$$

In these coordinates the extrinsic curvature tensor of the worldvolume at $y^{i}=0$ is

$$
K_{a b}^{i}=\Gamma_{a b}^{i}=-\Gamma_{a j}^{c} \eta_{c b} \delta^{j i}
$$

and is assumed to have a typical size

$$
K_{a b}^{i} \sim R^{-1}
$$

In the case of a particle, $p=0$, the extrinsic curvature corresponds to its acceleration, $a^{i}=K_{t t}^{i}$.

For $p$-branes with $p \geq 1$ there are additional terms of first order in derivatives,

$$
\omega_{a j}^{i}=\Gamma_{a j}^{i}=-\Gamma_{a k}^{l} \delta_{l j} \delta^{k i},
$$

which reflect the possibility of non-trivial holonomies along the worldvolume. Including them, the metric to first order in $y / R$ reads

$$
d s^{2}=\left(\eta_{a b}-2 K_{a b}^{i} y_{i}\right) d \sigma^{a} d \sigma^{b}+2 \omega_{a j}^{i} y_{i} d \sigma^{a} d y^{j}+d y_{i} d y^{i}+O\left(y^{2} / R^{2}\right)
$$

The coefficients $K_{a b}^{i}$ are components of a tensor and cannot be removed by a coordinate transformation. However, the transformation

$$
y^{i} \rightarrow y^{i}-\sigma^{a} \omega_{a j}^{i} y^{j}+O\left(\sigma^{2}\right)
$$

which is a $\sigma^{a}$-dependent rotation of the orthogonal directions, eliminates the $\omega_{a}{ }_{j}$ from the metric. So, locally, we can always set these to zero. Since our aim is to obtain local 
equations for the $p$-brane, such terms never enter our analysis and we can eliminate them. However, when one considers the global construction of a blackfold solution, attention must be paid to possible global obstructions. Such effects appear, for instance, in the study of blackfolds in Taub-NUT spaces [11].

We conclude that the metric in Fermi normal coordinates for a $p$-brane is, to first order,

$$
d s^{2}=\left(\eta_{a b}-2 K_{a b}{ }^{i} y_{i}\right) d \sigma^{a} d \sigma^{b}+d y_{i} d y^{i}+O\left(y^{2} / R^{2}\right) .
$$

This geometry characterizes completely the extrinsic metric perturbation to first derivative order, and can be regarded as the background that surrounds the black brane. The slowly fluctuating metric (2.2) under a generic extrinsic curvature perturbation takes the form

$$
\begin{aligned}
d s^{2}= & \left(\eta_{a b}-2 K_{a b}^{i} y_{i}+\frac{r_{0}^{n}}{r^{n}} u_{a} u_{b}\right) d \sigma^{a} d \sigma^{b}+\frac{d r^{2}}{1-\frac{r_{0}^{n}}{r^{n}}}+r^{2} d \Omega_{(n+1)}^{2} \\
& +h_{\mu \nu}\left(y^{i}\right) d x^{\mu} d x^{\nu}+O\left(r^{2} / R^{2}\right) .
\end{aligned}
$$

Here $r$ is the radial coordinate orthogonal to $\mathcal{W}_{p+1}$,

$$
r=\sqrt{y_{i} y^{i}}
$$

and $\Omega_{(n+1)}$ denotes the sphere at constant $r$ in the transverse direction, with direction cosines $y^{i} / r$. The metric corrections $h_{\mu \nu}$ depend on the parameters $r_{0}$ and $u^{a}$, and are linear in the $K_{a b}{ }^{i}$, hence they are of first order in $1 / R$.

We now make again use of our restriction to locally linearized fluctuations: the perturbations induced by the extrinsic curvature along each of the transverse directions $y^{i}$ decouple from each other. Thus we can deal with the deformation in each normal direction $i$ separately, and study the perturbations when $K_{a b}{ }^{i}$ is non-zero along only one direction $i=\hat{i}$. Introducing a direction cosine for $y^{\hat{i}}$ such that

$$
y^{\hat{i}}=r \cos \theta,
$$

then (2.12) becomes

$$
\begin{aligned}
d s^{2}= & \left(\eta_{a b}-2 K_{a b}{ }^{\hat{i}} r \cos \theta+\frac{r_{0}^{n}}{r^{n}} u_{a} u_{b}\right) d \sigma^{a} d \sigma^{b}+\frac{d r^{2}}{1-\frac{r_{0}^{n}}{r^{n}}}+r^{2} d \theta^{2}+r^{2} \sin ^{2} \theta d \Omega_{(n)}^{2} \\
& +h_{\mu \nu}(r, \theta) d x^{\mu} d x^{\nu}+O\left(r^{2} / R^{2}\right) .
\end{aligned}
$$

The extrinsic curvature deformations are proportional to $\cos \theta$, and therefore are dipoles of $S^{n+1}$. Since the different multipoles decouple from each other in a linearized analysis of perturbations, the corrections must also be dipoles and hence of the form 3

$$
h_{\mu \nu}(r, \theta)=\cos \theta \hat{h}_{\mu \nu}(r) \text {. }
$$

\footnotetext{
${ }^{3}$ Detailed arguments for these points have been presented in [12].
} 
One can also show that in this case $h_{\theta \theta}=h_{\Omega_{(n)} \Omega_{(n)}}$. In addition we can, and shall, choose a gauge in which $h_{r \theta}=0$.

In this way we have reduced the problem of finding the solution (2.15) of the Einstein equations to that of solving a set of coupled ordinary differential equations for functions $\hat{h}_{\mu \nu}(r)$ of the form

$$
\hat{h}_{\mu \nu}(r) d x^{\mu} d x^{\nu}=\hat{h}_{a b}(r) d \sigma^{a} d \sigma^{b}+\hat{h}_{r r}(r) d r^{2}+\hat{h}_{\Omega \Omega}(r)\left(d \theta^{2}+\sin ^{2} \theta d \Omega_{(n)}^{2}\right) .
$$

Some of the Einstein equations are actually constraints, and we turn to them first.

\section{Blackfold equations}

Let us focus on the asymptotic region of (2.15) at large $r \gg r_{0}$, where the gravitational field that the black brane creates is weak. If we expand (2.15) to linear order in $r_{0}^{n}$ we find

$$
\begin{aligned}
d s^{2}= & \left(\eta_{a b}-2 K_{a b}{ }^{\hat{i}} r \cos \theta+\frac{r_{0}^{n}}{r^{n}} u_{a} u_{b}\right) d \sigma^{a} d \sigma^{b}+\left(1+\frac{r_{0}^{n}}{r^{n}}\right) d r^{2}+r^{2}\left(d \theta^{2}+\sin ^{2} \theta d \Omega_{(n)}^{2}\right) \\
& +\cos \theta \hat{h}_{\mu \nu}(r) d x^{\mu} d x^{\nu}+O\left(r_{0}^{2 n} / r^{2 n}\right) .
\end{aligned}
$$

This can be written as

$$
\begin{aligned}
d s^{2}= & \left(\eta_{a b}-2 K_{a b}{ }^{\hat{i}} r \cos \theta+\frac{16 \pi G}{n \Omega_{(n+1)}}\left(T_{a b}-\frac{1}{D-2} T \eta_{a b}\right) \frac{1}{r^{n}}\right) d \sigma^{a} d \sigma^{b} \\
& +\left(1-\frac{16 \pi G}{\Omega_{(n+1)}} \frac{1}{D-2} \frac{T}{r^{n}}\right) d r^{2}+r^{2}\left(d \theta^{2}+\sin ^{2} \theta d \Omega_{(n)}^{2}\right)+ \\
& +\cos \theta \hat{h}_{\mu \nu}(r) d x^{\mu} d x^{\nu}+O\left(T_{a b}^{2} / r^{2 n}\right)
\end{aligned}
$$

where $T_{a b}$ is the stress-energy tensor (2.3) and $T=\eta^{a b} T_{a b}$. In fact, the asymptotic form of the metric (3.2) is generic for any gravitating $p$-brane, not necessarily a vacuum solution, when $T_{a b}$ is the brane stress-energy tensor (e.g., the quasilocal one) measured at $r \gg r_{0}$.4 By restricting the analysis to the large- $r$ asymptotic region we can be generic and consider $p$-branes with arbitrary $T_{a b}$, instead of only the case of neutral black $p$-branes that is studied in the rest of the paper. The above form of the metric assumes that the brane solution does not have any fields with stress-energy components along the directions transverse to the brane which would enter to the required order. We are effectively assuming that the brane is sufficiently localized in its transverse directions and that there are no external fields nor other forces acting on it. As a

\footnotetext{
${ }^{4}$ Leaving aside the extrinsic curvature perturbation, the asymptotic form of this metric is more often given in isotropic coordinates, which are obtained by changing $r \rightarrow r-16 \pi G T /\left(2 n(D-2) \Omega_{(n+1)} r^{n-1}\right)$.
} 
consequence, in this asymptotic region we have effectively vacuum equations, even for non-vacuum branes.

The corrections $\hat{h}_{\mu \nu}(r)$ in (3.2) are considered only to leading order in both $r / R$ and $T_{a b} / r^{n}$, and are obtained by solving Einstein's equations. By direct computation of the Einstein tensor $G_{\mu \nu}$ of (3.2), one finds that the combination

$$
G_{r \theta}-\frac{r \tan \theta}{n+1} G_{r r}=\frac{n+2}{n+1} \frac{\sin \theta}{r^{n}} \frac{8 \pi G}{\Omega_{(n+1)}} T^{a b} K_{a b} \hat{i}
$$

does not involve the $\hat{h}_{\mu \nu}$. Thus the corresponding Einstein equation is a constraint 5 which takes the form

$$
T^{a b} K_{a b}{ }^{\hat{i}}=0 .
$$

Here $\hat{i}$ denotes an arbitrary direction transverse to the brane, so these are the extrinsic equations of motion (1.2), derived for a generic gravitating $p$-brane curved in a manner governed by the Einstein equations.

It may be worth noting that if these equations are not satisfied, the geometry develops singularities on $\theta=0$ or $\theta=\pi$ at all $r$, reflecting the presence of unbalanced stresses. These singularities are possibly conical defects when $n=1$, like in the case of five-dimensional unbalanced black rings, but more generally they give rise to divergent curvatures. Note, however, that such singularities are unrelated to the possible presence of a black brane horizon.

In the next section we discuss the solution at all values of $r$, without requiring that $r \gg\left(T_{a b}\right)^{1 / n}$.

\section{Perturbed metric solution}

We return again to the particular case of a neutral black $p$-brane. We intend to solve for the perturbed geometry, in such a way that the horizon remains regular, for any extrinsic perturbation that satisfies (3.4) with the stress-energy tensor (2.3), i.e.,

$$
n u^{a} u^{b} K_{a b}^{\hat{i}}=K^{\hat{i}} .
$$

For the remainder of this section we shall omit the transverse index $\hat{i}$ from the extrinsic curvature in order to lighten the notation. Our analysis draws heavily on the one in [12, 13], which we simplify and reformulate in a worldvolume-covariant manner that applies to generic long-wavelength extrinsic perturbations of black $p$-branes.

\footnotetext{
${ }^{5}$ This must follow from the Gauss-Codacci equations for a surface at large constant $r$, but we have not investigated the precise relationship in detail.
} 
The metric corrections $\hat{h}_{\mu \nu}(r)$ in (2.17) are computed to linear order in the extrinsic curvature perturbation and therefore must be proportional to worldvolume tensor structures of order $1 / R$ built out of $K_{a b}, \eta_{a b}$ and $u^{a}$. They can be written in terms of only five functions, in the form

$$
\begin{gathered}
\hat{h}_{a b}(r)=K_{a b} \mathrm{~h}_{1}(r)+u^{c} u_{(a} K_{b) c} \mathrm{~h}_{2}(r)+K u_{a} u_{b} h_{\gamma}(r), \\
\hat{h}_{r r}(r)=K\left(1-\frac{r_{0}^{n}}{r^{n}}\right)^{-1} h_{r}(r), \\
\hat{h}_{\Omega \Omega}(r)=K r^{2} h_{\Omega}(r) .
\end{gathered}
$$

We have imposed eq. (4.1) in order to reduce the number of independent tensor structures. In particular, the only independent worldvolume scalar is $K$, and therefore $\hat{h}_{r r}$ and $\hat{h}_{\Omega \Omega}$ must be proportional to it. In $\hat{h}_{a b}$ we could have included a piece proportional to $K \eta_{a b}$. However, one can easily argue that this term must vanish. Consider a brane with directions in its worldvolume that are orthogonal to the extrinsic curvature. Terms $\propto \eta_{a b}$ deform the brane in these directions, but there cannot be any such deformations since there is no force to induce them - the dynamics along these directions can be consistently truncated out. It might seem that, by a similar argument, the function $h_{\gamma}(r)$ that multiplies $K u_{a} u_{b}$ should vanish, too. However, this term can be generated by a residual diffeomorphism. This is the freedom to change coordinates to first order in $K$ as

$$
r \rightarrow r+K \cos \theta \gamma(r), \quad \theta \rightarrow \theta+K \sin \theta \int^{r} d r^{\prime} \frac{\gamma\left(r^{\prime}\right)}{r^{\prime 2}\left(1-\frac{r_{0}^{n}}{r^{\prime \prime}}\right)}
$$

under which $h_{1}$ and $h_{2}$ remain invariant but

$$
\begin{aligned}
& h_{\gamma} \rightarrow h_{\gamma}-n \frac{r_{0}^{n}}{r^{n+1}} \gamma(r), \\
& h_{r} \rightarrow h_{r}+2 \gamma^{\prime}(r)-n \frac{r_{0}^{n}}{r^{n+1}} \frac{\gamma(r)}{1-\frac{r_{0}^{n}}{r^{n}}}, \\
& h_{\Omega}^{\prime} \rightarrow h_{\Omega}^{\prime}+2 \frac{\gamma^{\prime}(r)}{r}+2 \frac{r_{0}^{n}}{r^{n+2}} \frac{\gamma(r)}{1-\frac{r_{0}^{n}}{r^{n}}} .
\end{aligned}
$$

In the way we have written $\hat{h}_{r r}(r)$, in order that the horizon remains at $r=r_{0}$ these transformations are constrained to satisfy $\gamma\left(r_{0}\right)=0$.

We might use this freedom for, e.g., setting $h_{\gamma}$ to zero. However, since it will turn out that horizon regularity demands that $h_{\gamma}\left(r_{0}\right) \neq 0$, it is preferrable to leave it as a 
gauge-dependent variable and introduce two functions

$$
\begin{aligned}
\mathrm{h}_{r} & =h_{r}+\frac{2}{n} r_{0}\left(\frac{r^{n+1}}{r_{0}^{n+1}} h_{\gamma}\right)^{\prime}-\frac{h_{\gamma}}{1-\frac{r_{0}^{n}}{r^{n}}}, \\
\mathrm{~h}_{\Omega}^{\prime} & =h_{\Omega}^{\prime}+\frac{2}{n} \frac{r_{0}}{r}\left(\frac{r^{n+1}}{r_{0}^{n+1}} h_{\gamma}\right)^{\prime}+\frac{2}{n r} \frac{h_{\gamma}}{1-\frac{r_{0}^{n}}{r^{n}}}
\end{aligned}
$$

that are invariant under (4.5).

The general extrinsic perturbations are then characterized by the four gauge-invariant functions $\mathrm{h}_{1}, \mathrm{~h}_{2}, \mathrm{~h}_{r}$ and $\mathrm{h}_{\Omega}$, subject to suitable boundary conditions. In order to maintain the asymptotic behavior in (3.1) we require that $\hat{h}_{\mu \nu}(r)=O\left(r^{-n+1}\right)$ as $r \rightarrow \infty$. This constrains the residual gauge symmetry (4.5) to transformations with $\gamma^{\prime}=O\left(r^{-n+1}\right)$. For the gauge-invariant functions, the asymptotic behavior is then fixed to be

$$
\mathrm{h}_{1,2}=O\left(r^{-n+1}\right), \quad \mathrm{h}_{r, \Omega}=r^{2} O\left(r^{-1}\right) .
$$

In order to find the solution it suffices to solve the equations for some particular configuration of the extrinsic curvature and velocity that excites the three structures in (4.2). This has been done already in [12, 13, and in appendix $\mathrm{A}$ we give some details on how to convert the results to our set up.

Some of the solutions to the linear perturbation equations with the asymptotics (4.8), while satisfying the extrinsic blackfold equations (4.1), give singular behavior of $\mathrm{h}_{1,2}$ on the horizon and thus we discard them. The solution where $\mathrm{h}_{1,2}$ remain finite at $r=r_{0}$ and which satisfies (4.8) is unique and reads

$$
\begin{gathered}
\mathrm{h}_{1}=2 r-A P_{1 / n}\left(2 \frac{r^{n}}{r_{0}^{n}}-1\right), \\
\mathrm{h}_{2}=-A \frac{r_{0}^{n}}{r^{n}}\left[P_{1 / n}\left(2 \frac{r^{n}}{r_{0}^{n}}-1\right)+P_{-1 / n}\left(2 \frac{r^{n}}{r_{0}^{n}}-1\right)\right], \\
\mathrm{h}_{r}=\frac{n+1}{n^{2}\left(1-\frac{r_{0}^{n}}{r^{n}}\right)}\left[\left(\frac{n}{n+1}-2 \frac{r_{0}^{n}}{r^{n}}\right)\left(2 r-\mathrm{h}_{1}\right)-\mathrm{h}_{2}\right], \\
\mathrm{h}_{\Omega}^{\prime}=\frac{1}{n r\left(1-\frac{r_{0}^{n}}{r^{n}}\right)}\left(2 r-\mathrm{h}_{1}+\frac{n+2}{2 n} \mathrm{~h}_{2}\right),
\end{gathered}
$$

where $P_{ \pm 1 / n}(x)$ are Legendre functions and we have defined a constant

$$
A=2 r_{0} \frac{\Gamma\left(\frac{n+1}{n}\right)^{2}}{\Gamma\left(\frac{n+2}{n}\right)} .
$$


Having found these functions, the complete metric is specified by making a choice of the gauge-dependent function $h_{\gamma}(r)$. This must be subject to the asymptotic boundary condition 6

$$
h_{\gamma}=O\left(r^{-n+1}\right) .
$$

In the next section we shall see that the value of $h_{\gamma}$ at $r=r_{0}$ is constrained by the requirement of horizon regularity. Indeed, it must be fixed in order to cancel in $h_{r}$ and $h_{\Omega}^{\prime}$ the singular behavior that $\mathrm{h}_{r}$ and $\mathrm{h}_{\Omega}^{\prime}$ have at that point.

\section{Horizon regularity}

Now we exhibit Eddington-Finkelstein coordinates for the perturbed solution which make the horizon manifestly regular.

It is convenient to organize the worldvolume tensor structures into components parallel and orthogonal to the velocity. Using the orthogonal projector

$$
P_{a b}=\eta_{a b}+u_{a} u_{b},
$$

and always assuming that the constraint (4.1) is satisfied, the perturbed metric takes the form

$$
\begin{aligned}
d s^{2}= & {\left[\eta_{a b}+\frac{r_{0}^{n}}{r^{n}} u_{a} u_{b}+\left(K_{c d} P_{a}^{c} P_{b}^{d} \mathrm{~g}_{1}+u^{c} K_{c d} P_{a}^{d} u_{b} \mathrm{~g}_{2}+K u_{a} u_{b} g_{\gamma}\right) \cos \theta\right] d \sigma^{a} d \sigma^{b} } \\
& +\left(1+K h_{r} \cos \theta\right) \frac{d r^{2}}{1-\frac{r_{0}^{n}}{r^{n}}}+r^{2}\left(1+K h_{\Omega} \cos \theta\right)\left(d \theta^{2}+\sin ^{2} \theta d \Omega_{(n)}^{2}\right)
\end{aligned}
$$

where the new functions are obtained from the ones in the previous section as

$$
\begin{aligned}
& \mathrm{g}_{1}=\mathrm{h}_{1}-2 r, \\
& \mathrm{~g}_{2}=4 r-2 \mathrm{~h}_{1}+\mathrm{h}_{2}, \\
& g_{\gamma}=h_{\gamma}+\frac{1}{n}\left(\mathrm{~h}_{1}-\mathrm{h}_{2}-2 r\right) .
\end{aligned}
$$

The $\mathrm{g}_{1,2}$ are invariant under the gauge transformation (4.5), while $g_{\gamma}$ is changed by it. The extrinsic curvature terms of the asymptotic Fermi frame have been absorbed in these functions.

\footnotetext{
${ }^{6}$ In general this modifies the asymptotic Fermi normal frame by introducing terms in $h_{r r}, h_{\Omega \Omega}$ $\sim K r \cos \theta$. If we we want to avoid them we must require $h_{\gamma}=\frac{1}{2} \frac{r_{0}^{n}}{r^{n-1}}+O\left(r^{-1-n}\right)$.
} 
Expanding the solutions (4.9) around $r=r_{0}$, we find that

$$
\begin{aligned}
& \mathrm{g}_{1}=-A+O\left(r-r_{0}\right), \\
& \mathrm{g}_{2}=\frac{2 A(n+1)}{r_{0}}\left(r-r_{0}\right)+O\left(r-r_{0}\right)^{2}, \\
& g_{\gamma}=h_{\gamma}\left(r_{0}\right)+\frac{A}{n}+O\left(r-r_{0}\right), \\
& h_{r}=\left(h_{\gamma}\left(r_{0}\right)+\frac{A}{n}\right) \frac{r_{0}}{n\left(r-r_{0}\right)}+O\left(r-r_{0}\right)^{0}, \\
& h_{\Omega}^{\prime}=-\left(h_{\gamma}\left(r_{0}\right)+\frac{A}{n}\right) \frac{2}{n^{2}\left(r-r_{0}\right)}+O\left(r-r_{0}\right)^{0} .
\end{aligned}
$$

From the last two expressions it is apparent that in order to preserve regularity at $r=r_{0}$ it is necessary to set

$$
h_{\gamma}\left(r_{0}\right)=-\frac{A}{n},
$$

which also makes $g_{\gamma}$ vanish at $r_{0}$. This implies that $u^{a} u^{b} g_{a b}$ is zero there.

Condition (5.11) turns out to be sufficient for regularity. When it is imposed, one has

$$
\begin{aligned}
g_{\gamma} & =\left(\frac{A}{r_{0}} \frac{1-n-2 n^{2}}{n^{2}}+h_{\gamma}^{\prime}\left(r_{0}\right)\right)\left(r-r_{0}\right)+O\left(r-r_{0}\right)^{2}, \\
h_{r} & =-\frac{r_{0}}{n} g_{\gamma}^{\prime}\left(r_{0}\right)+O\left(r-r_{0}\right) .
\end{aligned}
$$

Also, $h_{\Omega}(r)=\int d r h_{\Omega}^{\prime}(r)$ is regular at $r=r_{0}$ for any value of the integration constant.

Performing a change to Eddington-Finkelstein coordinates in the form

$$
d v=-u_{a} d \sigma^{a}+\frac{d r}{1-\frac{r_{0}^{n}}{r^{n}}},
$$

the metric becomes

$$
\begin{aligned}
d s^{2}= & 2\left(1+K h_{r}\left(r_{0}\right) \cos \theta\right) d r d v+2 A \frac{n+1}{n} u^{c} K_{c d} P_{a}^{d} \cos \theta d \sigma^{a} d r \\
& +\left(P_{a b}-A K_{c d} P_{a}^{c} P_{b}^{d} \cos \theta\right) d \sigma^{a} d \sigma^{b} \\
& +r^{2}\left(1+K h_{\Omega}\left(r_{0}\right) \cos \theta\right)\left(d \theta^{2}+\sin ^{2} \theta d \Omega_{(n)}^{2}\right)+O\left(r-r_{0}\right)
\end{aligned}
$$

which is manifestly non-singular at $r=r_{0}$.

Since the perturbation is purely dipolar, neither the horizon temperature nor its entropy-density receive any corrections [12. The horizon velocity is also uncorrected, since the generators of the horizon are the orbits of $u^{a}$. The values of $h_{r}\left(r_{0}\right)$ and $h_{\Omega}\left(r_{0}\right)$ are gauge-dependent and unconstrained by regularity requirements. Both of them could be set to zero if desired. The only physical effect on the geometry of the horizon is the distortion of the worldvolume by the extrinsic curvature in directions orthogonal to $u^{a}$. 


\section{Complete corrected black brane geometry}

For reference and further use, we compile the results for all the perturbations, intrinsic and extrinsic, in a manifestly covariant form in the parallel and transverse directions.

The metric for the fluctuating, curved black $p$-brane takes the form of (2.2), with worldvolume metric

$$
\gamma_{a b}=\eta_{a b}-2 K_{a b}{ }^{i}(\sigma) r \cos \theta_{i}+O\left(r^{2} / R^{2}\right) .
$$

Here $\cos \theta_{i}=y_{i} / r$ are direction cosines in $S^{n+1}$ which parametrize the transverse directions and satisfy

$$
\cos \theta_{i} \cos \theta^{i}=1, \quad d\left(\cos \theta_{i}\right) d\left(\cos \theta^{i}\right)=d \Omega_{(n+1)}^{2} .
$$

The $n+2$ indices $i=p+1, \ldots, D$ are summed over, when repeated, in all these equations.

The $K_{a b}{ }^{i}(\sigma), r_{0}(\sigma)$ and $u^{a}(\sigma)$ must solve the blackfold equations

$$
\begin{gathered}
\dot{u}_{a}+\frac{1}{n+1} \vartheta u_{a}=\partial_{a} \ln r_{0}, \\
n K_{a b}{ }^{i} u^{a} u^{b}=K^{i},
\end{gathered}
$$

where

$$
\dot{u}_{a}=u^{b} D_{b} u_{a}, \quad \vartheta=D_{a} u^{a}
$$

are the acceleration and expansion of the effective fluid's velocity. Then, every solution to these equations determines completely (up to the gauge freedom discussed in the previous section) a set of 'bulk' corrections $h_{\mu \nu}$. We split these into fluid corrections (functions $f_{\mu \nu}$ ) and extrinsic corrections (functions $h_{\mu \nu}{ }^{i}$ ), such that the complete metric is

$$
\begin{aligned}
d s^{2}= & \left(\eta_{a b}-2 K_{a b}{ }^{i}(\sigma) r \cos \theta_{i}+\frac{r_{0}(\sigma)^{n}}{r^{n}} u_{a}(\sigma) u_{b}(\sigma)\right) d \sigma^{a} d \sigma^{b}+\frac{d r^{2}}{1-\frac{r_{0}(\sigma)^{n}}{r^{n}}}+r^{2} d \Omega_{(n+1)}^{2} \\
& +\left(f_{a b}(r)+h_{a b}{ }^{i}(r) \cos \theta_{i}\right) d \sigma^{a} d \sigma^{b}+2 f_{a r}(r) d \sigma^{a} d r \\
& +\left(f_{r r}(r)+h_{r r}{ }^{i}(r) \cos \theta_{i}\right) d r^{2}+h_{\Omega \Omega^{i}}{ }^{(}(r) \cos \theta_{i} d \Omega_{(n+1)}^{2} .
\end{aligned}
$$

The fluid fluctuations do not deform the $S^{n+1}$, hence $f_{\Omega \Omega}$ are zero. 
Fluid corrections. These have been derived in [4]. We write them as

$$
\begin{aligned}
& f_{a b}(r)=\vartheta u_{a} u_{b} \mathrm{f}_{1}(r)+\left(\sigma_{a b}+\frac{1}{p} \vartheta P_{a b}\right) \mathrm{f}_{2}(r), \\
& f_{a r}(r)=\vartheta u_{a} \mathrm{f}_{3}(r)+\dot{u}_{a} \mathrm{f}_{4}(r), \\
& f_{r r}(r)=\vartheta\left(1-\frac{r_{0}^{n}}{r^{n}}\right)^{-1} \mathrm{f}_{r}(r) .
\end{aligned}
$$

The derivatives of $r_{0}$ have been eliminated through the equations (6.3). Here $P_{a b}$ is the orthogonal projector (5.1),$\sigma_{a b}$ is the shear of the velocity flow,

$$
\sigma_{a b}+\frac{1}{p} \vartheta P_{a b}=P_{a}^{c} P_{b}^{d} D_{(c} u_{d)},
$$

and the radial functions are

$$
\begin{aligned}
\mathrm{f}_{1}(r) & =\frac{r_{0}}{n(n+1)}\left(2-(n+2) \frac{r_{0}^{n}}{r^{n}}\right) \ln \left(1-\frac{r_{0}^{n}}{r^{n}}\right) \\
\mathrm{f}_{2}(r) & =\frac{2 r_{0}}{n} \ln \left(1-\frac{r_{0}^{n}}{r^{n}}\right) \\
\mathrm{f}_{3}(r) & =\frac{r_{0}}{n+1} \frac{1}{1-\frac{r_{0}^{n}}{r^{n}}}\left[\left(\frac{n+1}{n} \frac{r_{0}^{n}}{r^{n}}-\frac{1}{n}\right) \ln \left(1-\frac{r_{0}^{n}}{r^{n}}\right)-\frac{r_{0}^{n}}{r^{n}}\left(n \frac{r_{*}}{r_{0}}+1\right)\right]+\delta_{n, 1}, \\
\mathrm{f}_{4}(r) & =\frac{r_{*}-r}{1-\frac{r_{0}^{n}}{r^{n}}}-\delta_{n, 1} r_{0} \ln \frac{r_{0}}{r}, \\
\mathrm{f}_{r}(r) & =\frac{r_{0}}{n+1} \frac{1}{1-\frac{r_{0}^{n}}{r^{n}}} \frac{r_{0}^{n}}{r^{n}}\left(2-\ln \left(1-\frac{r_{0}^{n}}{r^{n}}\right)\right),
\end{aligned}
$$

where

$$
r_{*}=\int \frac{d r}{1-\frac{r_{0}^{n}}{r^{n}}}={ }_{2} F_{1}\left(1,-\frac{1}{n} ; \frac{n-1}{n} ; \frac{r_{0}^{n}}{r^{n}}\right) r .
$$

Observe that the structure $u_{(a} \dot{u}_{b)}$ might have appeared in $f_{a b}$, but it turns out not to contribute.

Extrinsic corrections. These are the ones obtained in section 4. Now we simply include the index $i$ for all possible directions orthogonal to the worldvolume,

$$
\begin{aligned}
h_{a b}{ }^{i}(r) & =K_{a b}{ }^{i} \mathrm{~h}_{1}(r)+u^{c} u_{(a} K_{b) c}{ }^{i} \mathrm{~h}_{2}(r)+K^{i} u_{a} u_{b} h_{\gamma}(r), \\
h_{r r}{ }^{i}(r) & =K^{i}\left(1-\frac{r_{0}^{n}}{r^{n}}\right)^{-1} h_{r}(r), \\
h_{\Omega \Omega}{ }^{i}(r) & =K^{i} r^{2} h_{\Omega}(r),
\end{aligned}
$$

with the functions $\mathrm{h}_{1,2}, h_{r}, h_{\Omega}$ as given in (4.7), (4.9). The function $h_{\gamma}$ is only constrained to satisfy (4.11) and (5.11), and the integration constant in $h_{\Omega}$ can be chosen arbitrarily.

Eq. (5.2) gives an alternative form for the metric, with a decomposition of $g_{a b}$ based on directions parallel and orthogonal to $u^{a}$. 


\section{Discussion}

We have shown that the effective blackfold formalism of [2] can be fully derived from the Einstein equations for a deformed black brane, and results in geometries that are regular on and outside the black brane horizon. In particular this proves that all the new solutions in [3] are, to leading derivative order, bona fide black holes. In fact our solution applies to neutral black branes in any background with typical length scales much larger than $r_{0}$. This includes (Anti-)deSitter backgrounds with cosmological radius $\sqrt{|\Lambda|} \gg r_{0}$ and black hole backgrounds with horizon sizes $R_{h} \gg r_{0}$ [13, 14].

The extension to higher derivative orders is presumably technically complicated, since the different types of perturbations couple at the second and higher orders. Particular subsets of perturbations may be more amenable to study, but one should bear in mind that all the problems that one encounters in the analysis of self-force for small black holes [5] are probably exacerbated for extended objects like black $p$-branes.

Our study motivates the discussion of several issues:

The metric at all scales. Having obtained the solution in section 6 for the deformed black $p$-brane in the region where $r \ll R$, finding the metric valid at all radii only requires to complete a straightforward matched asymptotic expansion.

The construction is as follows. The black brane modifies the geometry of the background spacetime that it lives in. To first order for a thin brane, the correction is the linearized field sourced by a stress-energy tensor $T_{a b}$ localized on the worldvolume at $x^{\mu}=X^{\mu}\left(\sigma^{a}\right)$. Linearized gravity is a well studied subject and, in e.g., Minkowski or AdS spacetimes the required solution can be readily expressed in terms of integrals of known Green's functions over the given source.

The solution to this problem in linearized gravity gives the 'far zone' geometry, in the region where the brane thickness is negligible, $r \gg r_{0}$. At small $r$, we know that this must match the geometry (3.1) with specific values for $K_{a b}{ }^{i}$, since this form is valid for $r_{0} \ll r \ll R$ (the 'overlap zone'). The solution in section [6 extends this geometry into the entire region $r \ll R$ - the 'near zone' - for any $K_{a b}{ }^{i}$. So, once we make explicit the 'far zone' metric for the specific brane source, the results in this paper give the complete metric for the bent black brane.

Viscous fluid, elastic solid. The solution for the strained black brane in section 6 can be used to compute the stresses induced on the black brane as derivative corrections to $T_{a b}$. From these stress-strain relations we can extract linear-response coefficients, namely, the effective fluid viscosities and moduli of elasticity. These have been calculated in [4] and [15] respectively. We present the resulting stress-energy tensor for 
neutral black $p$-branes in the form

$$
T_{a b}=\left(\varepsilon u_{a} u_{b}+P P_{a b}-2 \eta \sigma_{a b}-\zeta \vartheta P_{a b}+\tilde{Y}_{a b}{ }^{c d} K_{c d}{ }^{i} \partial_{i}\right) \delta_{\perp}^{(n+2)}\left(x^{i}-X^{i}\left(\sigma^{a}\right)\right)
$$

where $\delta_{\perp}^{(n+2)}$ localizes in directions transverse to the worldvolume, and the constitutive relations are

$$
\begin{gathered}
\frac{\varepsilon}{n+1}=-P=\frac{\Omega_{(n+1)} r_{0}^{n}}{16 \pi G}, \\
\eta=\frac{\Omega_{(n+1)} r_{0}^{n+1}}{16 \pi G}, \quad \zeta=2 \eta\left(\frac{1}{p}+\frac{1}{n+1}\right),
\end{gathered}
$$

and

$$
\begin{aligned}
\tilde{Y}_{a b}{ }^{c d}= & \frac{\Omega_{(n+1)} r_{0}^{n}}{16 \pi G} \frac{n \tan (\pi / n)}{4 \pi} A^{2}\left(\frac{1}{n+2} \delta_{(a}{ }^{c} \delta_{b)}{ }^{d}+2 u_{(a} \delta_{b)}{ }^{(c} u^{d)}+\frac{3 n+4}{n+2} u_{a} u_{b} u^{c} u^{d}\right) \\
& +\tilde{k} \frac{n \tan (\pi / n)}{4 \pi} A^{2}\left[\left(\varepsilon u_{a} u_{b}+P P_{a b}\right) \eta^{c d}+\eta_{a b}\left(\varepsilon u^{c} u^{d}+P P^{c d}\right)\right] .
\end{aligned}
$$

The expression for the elasticity moduli $\tilde{Y}_{a b}{ }^{c d}$ is symmetric under $\{a b\} \leftrightarrow\{c d\}$ and differs from [15] by terms that vanish in $T_{a b}$ when the equations of motion are imposed. The free constant $\tilde{k}$ comes from the residual diffeomorphism (4.5) and parametrizes an ambiguity in the specification of the worldvolume surface.7

It is worth stressing that these values of the linear-response coefficients are fixed by the requirement of regularity of the horizon in the perturbed geometry, as imposed in sec. 4 and in [4].

Blackfold boundaries. Our analysis applies to black branes whose thickness $r_{0}$ remains finite. However, at blackfold boundaries the thickness vanishes [2]. The current evidence is that when this happens as a consequence of the velocity $u^{a}$ becoming lightlike, one obtains a regular horizon [1, 3, 16]. If, instead, it is due to the blackfold intersecting a horizon of the background, then a conifold-type singularity appears [17]. These phenomena remain the only aspect of the effective blackfold theory for which we still do not have a general understanding.

Other black branes and AdS/CFT. The effective theory of blackfolds in [2] has been extended to charged black branes [18, 16, 19], and for these, the analysis of

7 The dimensionally correct Young modulus $Y_{a b}{ }^{c d}$ as defined in [15] is

$$
Y_{a b}{ }^{c d}=\frac{(n+2)(n+4)}{r_{0}^{n+4}} \frac{\Omega_{(n)}}{\Omega_{(n+1)}} \tilde{Y}_{a b}^{c d},
$$

and is valid for $n>2$. The gauge $\tilde{k}_{2}=0$ for the dipole in [15] corresponds to $\tilde{k}=-\frac{(n+1)(n+4)}{n^{2}(n+2)}$ here. 
section 4 (but not that of section 3) needs to be redone since it involves a different set of field equations. We do not expect any difficulties of principle in doing it.

D-branes are a particular case of the charged branes analyzed with blackfold methods in [19], and one may study their bending by an extension of our approach. Some of these D-branes admit a decoupling limit in which the flat, extremal brane geometries become of type $\mathrm{AdS}_{p+2} \times S^{n+1}$, famously dual to conformal field theories. Extrinsic perturbations such as we have studied give rise to deformations of the $S^{n+1}$, and correspond to giving certain vev's for the scalar fields in the R-symmetry group (locally $S O(n+2))$ of the dual conformal theory. It may be of interest to compute this effect.

\section{Acknowledgments}

JC thanks the University of Barcelona for warm hospitality. We are also grateful to the String Theory Group at University of Amsterdam for hospitality in the last stages of this work, during the workshop on "Holographic Fluids". JC was supported by the STFC Consolidated Grant ST/J000426/1. RE was supported by MEC FPA201020807-C02-02, AGAUR 2009-SGR-168 and CPAN CSD2007-00042 Consolider-Ingenio 2010 .

\section{A Solution of Einstein's equations}

The results we presented in sec. 4 are the worldvolume covariantization of the solutions in [12, 13]. In this appendix we give some details on how the functions $\mathrm{h}$ of sec. 4 are related to those in [12, 13].

Refs. [12, 13] study the case $p=1$, with a boost parametrized by rapidity $\alpha$ such that $u_{a}=\left(-c_{\alpha},-s_{\alpha}\right)$, and with extrinsic curvature $K_{a b}=\operatorname{diag}\left(C_{t},-C_{z}\right)$. In this case the other tensor structure in eqs. (4.2) is

$$
u^{c} u_{(a} K_{b) c}=-\left(\begin{array}{cc}
C_{t} c_{\alpha}^{2} & \frac{C_{t}+C_{z}}{2} c_{\alpha} s_{\alpha} \\
\frac{C_{t}+C_{z}}{2} c_{\alpha} s_{\alpha} & C_{z} s_{\alpha}^{2}
\end{array}\right) .
$$

The functions A and B in [12, 13] are related to our $\hat{h}_{a b}$ as

$$
\begin{aligned}
\mathrm{A} & =\hat{h}_{t t}-\frac{c_{\alpha}^{2}}{s_{\alpha}^{2}} \hat{h}_{z z} \\
& =\left(K_{t t}-\frac{c_{\alpha}^{2}}{s_{\alpha}^{2}} K_{z z}\right)\left(\mathrm{h}_{1}-2 r\right)+\left(u^{c} u_{t} K_{t c}-\frac{c_{\alpha}^{2}}{s_{\alpha}^{2}} u^{c} u_{z} K_{z c}\right) \mathrm{h}_{2} \\
& =\left(C_{t}+\frac{c_{\alpha}^{2}}{s_{\alpha}^{2}} C_{z}\right)\left(\mathrm{h}_{1}-2 r\right)+c_{\alpha}^{2}\left(-C_{t}+C_{z}\right) \mathrm{h}_{2}
\end{aligned}
$$




$$
\begin{aligned}
\mathrm{B} & =\frac{1}{c_{\alpha} s_{\alpha}} \hat{h}_{t z}-\frac{1}{s_{\alpha}^{2}} \hat{h}_{z z} \\
& =\left(\frac{1}{c_{\alpha} s_{\alpha}} K_{z z} K_{t z}-\frac{1}{s_{\alpha}^{2}} K_{z z}\right)\left(\mathrm{h}_{1}-2 r\right)+\left(\frac{1}{c_{\alpha} s_{\alpha}} u^{c} u_{(t} K_{z) c}-\frac{1}{s_{\alpha}^{2}} u^{c} u_{z} K_{z c}\right) \mathrm{h}_{2} \\
& =\frac{C_{z}}{s_{\alpha}^{2}}\left(\mathrm{~h}_{1}-2 r\right)+\frac{C_{z}-C_{t}}{2} \mathrm{~h}_{2} .
\end{aligned}
$$

The function $h_{\gamma}$ does not appear here, which is equivalent to absence of the gaugedependent function $c(r)$ in [12, 13].

Refs. [12, 13] solved the linearized equations for a generic dipole perturbation of a black string and obtained four independent solutions. Two of them diverge at $r=r_{0}$ and are discarded, and the ones that remain finite at $r=r_{0}$ are

$$
\begin{aligned}
& u_{1}={ }_{2} F_{1}\left(-\frac{1}{n},-\frac{n+1}{n} ; 1 ; 1-\frac{r_{0}^{n}}{r^{n}}\right) r, \\
& u_{2}={ }_{2} F_{1}\left(-\frac{1}{n}, \frac{n-1}{n} ; 1 ; 1-\frac{r_{0}^{n}}{r^{n}}\right) \frac{r_{0}^{n}}{r^{n-1}} .
\end{aligned}
$$

In terms of these, the solution for $h_{1}$ and $h_{2}$ that satisfies the asymptotic boundary conditions (4.8) is

$$
\mathrm{h}_{1}=-\frac{A}{r_{0}} \frac{n+1}{n+2}\left(u_{1}+\frac{1}{n+1} u_{2}\right)+2 r, \quad \mathrm{~h}_{2}=-\frac{2 A}{r_{0}} u_{2},
$$

where $A$, defined in (4.10), gives a simpler form for the coefficient of $u_{1}$ than the constant $A_{1}$ in [12, 13]. It is straightforward to check that substituting (A.5) in (A.2) we recover the correct expressions for $A$ and $B$. We omit the details of how the functions $\mathrm{h}_{r}$ and $\mathrm{h}_{\Omega}^{\prime}$ are similarly obtained from $\mathrm{F}$ and $\mathrm{G}^{\prime}$ in [12, 13].

The hypergeometric functions in the solution are actually Legendre functions $P_{\nu}(x)$ with index $\nu= \pm 1 / n$ and argument $x=2\left(r / r_{0}\right)^{n}-1$. A simple example of such relations is

$$
{ }_{2} F_{1}\left(-\frac{1}{n},-\frac{1}{n} ; 1 ; 1-\frac{r_{0}^{n}}{r^{n}}\right)=\frac{r_{0}}{r} P_{1 / n}\left(2 \frac{r^{n}}{r_{0}^{n}}-1\right) .
$$

This and other similar expressions allow to rewrite the $\mathrm{h}$ functions as in (4.9). The Legendre functions $P_{\nu}(x)$ become Legendre polynomials when $\nu \in \mathbb{N}$, which in the present instance can only be when $n=1$.

\section{References}

[1] R. Emparan, T. Harmark, V. Niarchos and N. A. Obers, "Worldvolume Effective Theory for Higher-Dimensional Black Holes (Blackfolds)," Phys. Rev. Lett. 102, 191301 (2009) [arXiv:0902.0427 [hep-th]]. 
[2] R. Emparan, T. Harmark, V. Niarchos and N. A. Obers, "Essentials of Blackfold Dynamics," JHEP 1003 (2010) 063 [arXiv:0910.1601 [hep-th]].

[3] R. Emparan, T. Harmark, V. Niarchos and N. A. Obers, "New Horizons for Black Holes and Branes," JHEP 1004 (2010) 046. arXiv:0912.2352 [hep-th]].

[4] J. Camps, R. Emparan and N. Haddad, "Black Brane Viscosity and the GregoryLaflamme Instability," JHEP 1005 (2010) 042 [arXiv:1003.3636 [hep-th]].

[5] E. Poisson, A. Pound and I. Vega, "The Motion of point particles in curved spacetime," Living Rev. Rel. 14 (2011) 7 arXiv:1102.0529 [gr-qc]].

[6] B. Carter, "Essentials of classical brane dynamics," Int. J. Theor. Phys. 40 (2001) 2099 gr-qc/0012036.

[7] S. Bhattacharyya, V. E. Hubeny, S. Minwalla and M. Rangamani, "Nonlinear Fluid Dynamics from Gravity," JHEP 0802 (2008) 045 arXiv:0712.2456 [hepth]].

[8] R. C. Myers, "Stress tensors and Casimir energies in the AdS / CFT correspondence," Phys. Rev. D 60 (1999) 046002 [hep-th/9903203].

[9] J. D. Brown and J. W. York, Jr., "Quasilocal energy and conserved charges derived from the gravitational action," Phys. Rev. D 47 (1993) 1407 [gr-qc/9209012.

[10] B. Carter, "Outer curvature and conformal geometry of an imbedding," J. Geom. Phys. 8 (1992) 53.

[11] J. Camps, R. Emparan, P. Figueras, S. Giusto and A. Saxena, "Black Rings in Taub-NUT and D0-D6 interactions," JHEP 0902 (2009) 021 arXiv:0811.2088 [hep-th]].

[12] R. Emparan, T. Harmark, V. Niarchos, N. A. Obers and M. J. Rodríguez, "The Phase Structure of Higher-Dimensional Black Rings and Black Holes," JHEP 0710 (2007) 110 arXiv:0708.2181 [hep-th]].

[13] M. M. Caldarelli, R. Emparan and M. J. Rodriguez, "Black Rings in (Anti)deSitter space," JHEP 0811 (2008) 011 [arXiv:0806.1954 [hep-th]].

[14] J. Armas and N. A. Obers, "Blackfolds in (Anti)-de Sitter Backgrounds," Phys. Rev. D 83 (2011) 084039 [arXiv:1012.5081 [hep-th]].

[15] J. Armas, J. Camps, T. Harmark and N. A. Obers, "The Young Modulus of Black Strings and the Fine Structure of Blackfolds," arXiv:1110.4835 [hep-th]. 
[16] M. M. Caldarelli, R. Emparan and B. Van Pol, "Higher-dimensional Rotating Charged Black Holes," JHEP 1104 (2011) 013 [arXiv:1012.4517 [hep-th]].

[17] R. Emparan and N. Haddad, "Self-similar critical geometries at horizon intersections and mergers," JHEP 1110 (2011) 064 [arXiv:1109.1983 [hep-th]].

[18] G. Grignani, T. Harmark, A. Marini, N. A. Obers and M. Orselli, "Heating up the BIon," JHEP 1106 (2011) 058 [arXiv:1012.1494 [hep-th]].

[19] R. Emparan, T. Harmark, V. Niarchos and N. A. Obers, "Blackfolds in Supergravity and String Theory," JHEP 1108 (2011) 154 [arXiv:1106.4428 [hep-th]]. 